\title{
Serial multiple mediation of the association between internet gaming disorder and suicidal ideation by insomnia and depression in adolescents in Shanghai, China
}

Yuelin $\mathrm{Yu}^{1 \dagger}$, Xue Yang ${ }^{2 \dagger}$, Suping Wang ${ }^{1 \dagger}$, Huwen Wang ${ }^{1}$, Ruijie Chang ${ }^{1}$, Lhakpa Tsamlag ${ }^{1}$, Shuxian Zhang ${ }^{1}$, Chen $\mathrm{Xu}^{1}$, Xiaoyue $\mathrm{Yu}^{1}$, Yong Cai ${ }^{*}$ (i) and Joseph T. F. Lau ${ }^{2^{*}}$

\begin{abstract}
Background: High prevalence and strong relationships among suicidal ideation, Internet gaming disorder (IGD), insomnia, and depression have been reported for adolescents worldwide, but the mechanism underlying these psychological problems remains unclear. This cross-sectional study explored the mediating effect of insomnia and depression on the association between IGD and suicidal ideation.

Methods: Participants were 1066 adolescents (median age $=13.0$ years) with Internet games exposure in the previous 12 months from junior high schools in Shanghai, China. Questionnaire measures of suicidal ideation, IGD, insomnia, depressive symptoms, and background characteristics were obtained. Path analysis was conducted to test the multiple mediating roles of insomnia and depression.

Results: Suicidal ideation, IGD, insomnia, and depression prevalence was 27.2, 13.6, 9.2, and 17.0\%, respectively. A serial multiple mediation model was generated. The mediation effect of insomnia and depression on the pathway from IGD to suicidal ideation was 45.5\% (direct effect: standardized estimate [Std. estimate] = 0.186; total indirect effect: Std. estimate $=0.155$ ). The association between IGD and depression was partially mediated by insomnia (direct effect: Std. estimate $=0.211$; indirect effect: Std. estimate $=0.135$ ). The proposed model fit the data well.

Conclusions: Insomnia and depression may serially mediate the association between IGD and suicidal ideation. IGD was positively associated with insomnia, then with depression, which in turn positively contributed to suicidal ideation. We suggest greater monitoring of Internet use and prevention of insomnia and depression to mitigate the risk of suicidal ideation among Chinese adolescents.
\end{abstract}

Keywords: Internet gaming disorder, Insomnia, Depression, Suicidal ideation, Mediation

\footnotetext{
* Correspondence: caiyong202028@hotmail.com; jlau@cuhk.edu.hk

${ }^{\dagger}$ Yuelin Yu, Xue Yang and Suping Wang contributed equally to this work and should be considered co-first authors.

${ }^{1}$ School of Public Health, School of Medicine, Shanghai Jiao Tong University, Shanghai 200025, PR China

${ }^{2}$ Centre for Health Behaviors Research, JC School of Public Health and Primary Care, the Chinese University of Hong Kong, Hong Kong 810016, SAR, China
}

C C The Author(s). 2020 Open Access This article is licensed under a Creative Commons Attribution 4.0 International License, which permits use, sharing, adaptation, distribution and reproduction in any medium or format, as long as you give appropriate credit to the original author(s) and the source, provide a link to the Creative Commons licence, and indicate if changes were made. The images or other third party material in this article are included in the article's Creative Commons licence, unless indicated otherwise in a credit line to the material. If material is not included in the article's Creative Commons licence and your intended use is not permitted by statutory regulation or exceeds the permitted use, you will need to obtain permission directly from the copyright holder. To view a copy of this licence, visit http://creativecommons.org/licenses/by/4.0/ The Creative Commons Public Domain Dedication waiver (http://creativecommons.org/publicdomain/zero/1.0/) applies to the data made available in this article, unless otherwise stated in a credit line to the data. 


\section{Background}

Adolescence is one of the life stages that exhibits higher prevalence of suicides [1] and having suicide as the second leading cause of death (U.S. CDC). Suicidal ideation predicts subsequent suicidal plans, attempts, and behaviors [2, 3]. The global 12-month and lifetime prevalence for adolescent suicidal ideation was 14.2 and $18 \%$, respectively [4]. The prevalence of suicidal ideation has increased in Chinese adolescents. For instance, it increased from 10.4 to $13.6 \%$ among grade 8-9 Hong Kong Chinese students according to a 1year longitudinal study [5]. Given the rising prevalence and harmful consequences of suicidal ideation, as well as a tendency for suicidal ideation to appear at younger ages [6], early identification and effective intervention are warranted to reduce adolescent suicidal ideation and its risk factors.

Recent research on suicidal ideation has investigated adolescents with Internet gaming disorder (IGD). IGD is a another worldwide public health problem and has been included in ICD-11 in 2018 [7]. The overall global prevalence of adolescent IGD was 6.0\% [8], and was highest in east Asian countries [9]. IGD was associated with psychological disorders such as insomnia [10,11], depressive symptoms $[12,13]$, loneliness, anxiety [14], and suicidal ideation [1517]. A body of studies have shown that people addicted to internet would have a higher risk of suicidal ideation [18]. A dose-effect relationship was found between Internet game exposure of $\geq 5 \mathrm{~h}$ per day and suicidal ideation (odds ratio $[\mathrm{OR}]=1.7$; 95\% confidence interval [CI]: 1.3-2.1) [19]. Yet, few studies have explored the psychological factors that lead from IGD to suicidal ideation, and the mechanism underlying this association remains unclear.

The substantial association between both IGD and suicidal ideation and psychiatric problems such as insomnia and depression may shed insights to our understanding about the mechanism. Depression is one of the strongest predictors of suicidal ideation [20]. Majority (>90\%) of people who committed suicide express depressive symptoms simultaneously, and this co-occurrence rate in adolescent suicide cases is more than 50\% [21]. Similarly, adolescents with insomnia problems are more likely to generate suicidal ideation. One recent meta-analysis identified insomnia as a predictor of suicidal ideation $(\mathrm{OR}=2.35$; $95 \%$ CI: 1.58-1.92) [22]. Systematic reviews have also shown that insomnia $(\mathrm{OR}=2.20$; 95\% CI: 1.77-2.74) [23] and depression $(\mathrm{OR}=1.22 ; 95 \% \mathrm{CI}: 1.10-1.34)$ [24] are among the strongest correlates of IGD. A recent study indicates that depression mediates the association between addiction to mobile phone use and suicidality in Chinese adolescents (95\% CI: 27.891-69.831) [25]. Given their strong association with both IGD and suicidal ideation, insomnia and depression may be key mediators in the relationship between IGD and suicidal ideation. If so, this would help to narrow the causal gap between IGD and suicidal ideation.
Previous studies have suggested that insomnia can potentially mediate the relationship between IGD and depression, indicating that insomnia and depression may sequentially mediate the effect of IGD on suicidal ideation among adolescents. One meta-analysis of thirty-four cohort studies have revealed that insomnia serves as a strong predictor on depression [26]. There is a bidirectional causal relationship between IGD and insomnia; however, insomnia accounts for a greater proportion of the total effect on depression [27-29]. Thus, insomnia is accepted as a mediator on the relationship between IGD and depression. As there were strong associations as well as strong predictor effects between IGD, insomnia, depression and suicidal ideation, the following mediation model may be implied that adolescents with IGD may experience insomnia before developing depressive symptoms and, consequently, develop a high risk of suicidal ideation.

Although research suggests separate robust associations among psychological disorders, theoretical models are needed to explain how IGD affects suicidal ideation. Therefore, this study investigated a serial multiple mediation model based on previous study findings. The aim was [1] to investigate the prevalence for suicidal ideation, IGD, insomnia, and depression among adolescents in junior high school in Shanghai, China [2]; to test the association between suicidal ideation, IGD, insomnia, and depression [3]; to explore the serial multiple mediation effect of insomnia and depression on the association between IGD and suicidal ideation.

\section{Method \\ Participants and recruitment}

Using convenience sampling, six junior high schools from two districts of Shanghai, China, were selected from April to May 2018. All first-grade students (a total of 1329) from the selected schools were recruited. After removing observations with over $5 \%$ of items missing and low credibility, 1243 observations remained. Data from 1066 (85.8\%) students who self-reported themselves as Internet gamers in the past 12 months were used for statistical analysis.

Permission for this in-school survey was obtained before the investigation from schools, legal guardians, and students. As school principals are responsible for students, these were first informed about the study and their consent obtained. Then students and their legal guardians were informed about the study aims and procedure. Verbal informed consent was obtained from legal guardians and students themselves as they participated in the investigation during school time. Participants were informed that all data collected would be used only for research purposes and would be strictly confidential. The background, aim, procedure, and confidentiality of the study were explained at the top of the questionnaire. Participants were free to terminate their participation at any time with no adverse 
consequences. All eligible participants were asked to complete an anonymous structured questionnaire in class.

\section{Measures \\ Background characteristics}

The following background characteristics were analyzed: sex, age, mother's educational level, father's educational level, perceived family financial condition, residence identity (local or migrant residents), family type (single-parent family or not), and living arrangements (lives with parents or not). These background characteristics were selected by referring to the literature.

\section{Suicidal ideation}

Participants were asked how often over the last 12 months they had considered suicide. The three possible response options reflected the frequency of emerging suicidal ideation: "0" (never), "1" (once or twice), and " 2 " (more than twice). We categorized respondents into two groups for descriptive statistical analysis and logistic regression. Participants who chose "1" or "2" were deemed as exhibiting suicidal ideation and those who chose "0" were considered to show no suicidal ideation. Suicidal ideation category scale scores were used for path analysis.

\section{Internet gaming disorder (IGD)}

IGD was assessed using the diagnostic criteria in the Diagnostic and Statistical Manual of Mental Disorders Fifth Edition (DSM-V) [30]. The measure comprised nine items that assessed IGD symptoms. Participants rated how often they had experienced the symptoms in the previous 12 months on a yes/no scale; " 0 " indicated absence of the symptom and " 1 " indicated its presence. Positive responses on $\geq 5$ criteria were considered to indicate IGD (Cronbach's $\alpha=0.746)$. IGD continuous scale scores were used for path analysis.

\section{Insomnia}

Insomnia was assessed using the Insomnia Severity Index (ISI) [31]. The ISI is a 7-item self-report instrument that measures symptoms and insomnia-related problems. The scale has been validated and is widely used in insomnia studies [32-34]. The total ISI score ranges from 0 to 28; higher scores indicate more severe insomnia. Scores of 0-7 indicate no insomnia, 8-14 indicate subclinical insomnia, 15-21 indicate moderate clinical insomnia, and 22-28 indicate severe clinical insomnia (Cronbach's $\alpha=0.838$ ). Participants with a total score $>14$ are deemed to have clinical insomnia [35]. ISI continuous scale scores were used for path analysis.

\section{Depression}

The 9-item Patient Health Questionnaire (PHQ-9) [36] was used to evaluate depression, as many previous studies indicate its effectiveness and superiority for assessing depression [37, 38]. Total PHQ-9 scores range from 0 to 27; higher scores indicate more severe depression. Scores of 0-4 indicate no depression, 5-9 indicate mild depression, 10-14 indicate moderate depression, 15-19 indicate moderately severe depression, and 20-27 indicate severe depression (Cronbach's $\alpha=0.870$ ). Total scores $\geq 10$ are considered to indicate depression [39]. PHQ-9 continuous scale scores were used for path analysis.

\section{Statistical analysis}

Descriptive analyses were first conducted of background characteristics and the prevalence of suicidal ideation, IGD, insomnia, and depression. As the distribution of age was skewed, this continuous variable was described using the median (interquartile range [IQR]), and the median was used to divide this variable into two categories for the subsequent logistic regression. Categorical variables (suicidal ideation, IGD, insomnia level, depression level, sex, father's educational level, mother's educational level, perceived family financial condition, residence identity, family type, and living arrangements) were described using frequencies (percentages).

Univariate logistic regression was then performed to examine the association between background characteristics and suicidal ideation, and the association between psychological variables (IGD, insomnia, and depression) and suicidal ideation. After controlling statistically significant background characteristics, we included IGD, insomnia, and depression into a logistic regression model to obtain adjusted ORs (AORs) and the corresponding CIs. Moreover, pairwise correlation analysis of measurements (DSM$\mathrm{V}$ for IGD, ISI for insomnia, and PHQ-9 for depression, questionnaire for suicidal ideation) was used to test the relationships among the variables.

The serial multiple mediation hypothesis for IGD, insomnia, depression, and suicidal ideation was tested using Preacher and Hayes's method [40]. Bootstrapping analysis with 5000 resamples was conducted to test the significance of the mediation effects [41]. The weighted least squares and mean and variance estimator was used as the outcome was categorical. The significant background variable of suicidal ideation reported in the regression analysis was controlled. Model fit indices (root mean square error of approximation [RMSEA], comparative fit index [CFI], Tucker-Lewis index [TLI], standardized root mean square residual [SRMR]) were calculated to assess the model goodness of fit. RMSEA and SRMR values $<0.08$, and CFI and TLI values $>0.90$, indicated acceptable goodness of fit [42].

We used IBM SPSS Statistics 24.0 (IBM Corp., Armonk, NY, USA) to conduct the descriptive analysis, logistic regression, and pairwise correlation analysis, and used Mplus Version 8.3 (Muthen \& Muthen, Los Angeles, CA, USA) to conduct the path analysis. $P$ values $<0.05$ were considered statistically significant. 


\section{Results}

\section{Descriptive analyses}

As shown in Tables 1 and 2, 1,066 participants (median age $[\mathrm{IQR}]=13.0[12.0,13.0]$ years) had been exposed to Internet games in the previous 12 months; $43.5 \%$ were female. Most participants' parents (83.3\% mother; $85.8 \%$ father) had at least senior high school education. Over $90 \%$ of participants perceived their family financial condition as above or equivalent to medium, and $11.4 \%$ of participants were living in single-parent families.

Table 1 Descriptive and univariate logistic regression analysis of background characteristics on suicidal ideation ( $\geq$ once)

\begin{tabular}{|c|c|c|c|}
\hline \multirow[t]{2}{*}{ Characteristics } & \multirow{2}{*}{$\begin{array}{l}\text { Number of } \\
\text { participants }\end{array}$} & \multicolumn{2}{|c|}{ Suicidal Ideation( $\geq$ once) } \\
\hline & & $n($ row $)$ & ORu $(95 \% \mathrm{Cl})$ \\
\hline \multicolumn{4}{|l|}{ Sex (\%) } \\
\hline Male & $602(56.5 \%)$ & $121(20.1 \%)$ & Ref \\
\hline Female & $464(43.5 \%)$ & $169(36.4 \%)$ & $2.277(1.730,2.997) * * *$ \\
\hline Age (years), median (IQR) & $13.0(12.0,13.0)$ & & \\
\hline$<=12$ years $(\%)$ & $342(32.1 \%)$ & $107(31.3 \%)$ & Ref \\
\hline$>=13$ years $(\%)$ & $724(67.9 \%)$ & $183(25.3 \%)$ & $0.743(0.560,0.986) *$ \\
\hline \multicolumn{4}{|l|}{ Mother's education level (\%) } \\
\hline Primary school or below & $31(2.9 \%)$ & $13(41.9 \%)$ & Ref \\
\hline Junior high school & $124(11.7 \%)$ & $45(36.3 \%)$ & $0.789(0.354,1.758)$ \\
\hline Senior high school & $219(20.7 \%)$ & 59 (26.9\%) & $0.511(0.236,1.106)$ \\
\hline University or above & $664(62.6 \%)$ & $162(24.4 \%)$ & $0.447(0.214,0.932) *$ \\
\hline Unknown & $22(2.1 \%)$ & $10(45.5 \%)$ & $1.154(0.384,3.471)$ \\
\hline \multicolumn{4}{|l|}{ Father's education level (\%) } \\
\hline Primary school or below & $25(2.4 \%)$ & $10(40.0 \%)$ & Ref \\
\hline Junior high school & $112(9.6 \%)$ & $39(38.2 \%)$ & $0.929(0.380,2.271)$ \\
\hline Senior high school & $222(20.9 \%)$ & $68(30.6 \%)$ & $0.662(0.283,1.549)$ \\
\hline University or above & $689(64.9 \%)$ & $162(23.5 \%)$ & $0.461(0.203,1.046)$ \\
\hline Unknown & $23(2.2 \%)$ & $10(43.5 \%)$ & $1.154(0.366,3.640)$ \\
\hline \multicolumn{4}{|c|}{ Perceived family financial condition (\%) } \\
\hline Very good/good & $655(61.5 \%)$ & $144(22.0 \%)$ & Ref \\
\hline Medium & $318(29.9 \%)$ & $106(33.3 \%)$ & $1.774(1.318,2.389) * * *$ \\
\hline Very poor/poor & $26(2.4 \%)$ & $16(61.5 \%)$ & $5.678(2.522,12.781)^{* * *}$ \\
\hline Unknown & $66(6.2 \%)$ & $24(36.4 \%)$ & $2.028(1.188,3.461) * *$ \\
\hline \multicolumn{4}{|l|}{ Residence identity (\%) } \\
\hline Local & $881(82.8 \%)$ & $247(28.0 \%)$ & Ref \\
\hline Migrant & $183(17.2 \%)$ & $42(23.0 \%)$ & $0.765(0.526,1.112)$ \\
\hline \multicolumn{4}{|l|}{ Family type (\%) } \\
\hline Non single-parent family & $941(88.6 \%)$ & $249(26.5 \%)$ & Ref \\
\hline Single-parent family & $121(11.4 \%)$ & $41(33.9 \%)$ & $1.424(0.952,2.132)$ \\
\hline \multicolumn{4}{|l|}{ Living arrangements (\%) } \\
\hline Live with parents & $906(85.2 \%)$ & $237(26.2 \%)$ & Ref \\
\hline Only live with mother & $77(7.2 \%)$ & $25(32.5 \%)$ & $1.357(0.824,2.236)$ \\
\hline Only live with father & $37(3.5 \%)$ & $13(35.1 \%)$ & $1.529(0.766,3.052)$ \\
\hline Live with neither & $43(4.0 \%)$ & $14(32.6 \%)$ & $1.363(0.708,2.623)$ \\
\hline \multicolumn{4}{|l|}{ Suicidal ideation (\%) } \\
\hline Never think about it & $776(72.8 \%)$ & - & \\
\hline Think it more than once & $290(27.2 \%)$ & - & \\
\hline
\end{tabular}


Table 2 Descriptive and logistic regression analysis of psychological variables on suicidal ideation ( $\geq$ once)

\begin{tabular}{|c|c|c|c|c|}
\hline \multirow[t]{2}{*}{ Characteristics } & \multirow{2}{*}{$\begin{array}{l}\text { Number of } \\
\text { participants }\end{array}$} & \multicolumn{3}{|c|}{ Suicidal Ideation( $\geq$ once) } \\
\hline & & n(row\%) & ORu $(95 \% \mathrm{Cl})$ & AOR $(95 \% \mathrm{Cl})$ \\
\hline \multicolumn{5}{|l|}{ Internet gaming disorder (\%) } \\
\hline No & $921(86.4 \%)$ & $222(24.1 \%)$ & Ref & Ref \\
\hline Yes & $145(13.6 \%)$ & $68(46.9 \%)$ & $2.781(1.941,3.983) * * *$ & $3.089(2.100,4.544) * * *$ \\
\hline \multicolumn{5}{|l|}{ Insomnia condition (\%) } \\
\hline None insomnia (0-7) & $663(62.2 \%)$ & $113(17.0 \%)$ & Ref & Ref \\
\hline Subclinical insomnia [8-14] & $305(28.6 \%)$ & $122(40.0 \%)$ & $3.245(2.390,4.405) * * *$ & $3.052(2.224,4.189) * * *$ \\
\hline Clinical insomnia [14-28] & $98(9.2 \%)$ & $55(56.1 \%)$ & $6.226(3.980,9.738) * * *$ & $5.751(3.614,9.152) * * *$ \\
\hline \multicolumn{5}{|l|}{ Depression (\%) } \\
\hline None depression (0-4) & $556(52.2 \%)$ & $58(10.4 \%)$ & Ref & Ref \\
\hline Mild depression [5-9] & $329(30.9 \%)$ & $112(34.0 \%)$ & $4.432(3.108,6.320) * * *$ & $4.289(2.985,6.162) * * *$ \\
\hline Presence of depression [10-27] & $181(17.0 \%)$ & $120(66.3 \%)$ & $16.891(11.198,25.477)^{* * *}$ & $14.805(9.685,22.630)^{* * *}$ \\
\hline
\end{tabular}

$O R u$ Univariate odds ratio, $A O R$ Adjusted odds ratio for background characteristics included in this study

$\mathrm{Cl}$ Confidence interval

${ }^{*} p<0.05,{ }^{* *} p<0.01, * * * p<0.001$

Of adolescent Internet game players, the prevalence of suicidal ideation, IGD, clinical insomnia, and depression was 27.2, 13.6, 9.2, and $17.0 \%$, respectively. Participants with more severe levels of psychological disorder had greater suicidal ideation. About half of adolescents with IGD (46.9\%), clinical insomnia (56.1\%), and depression (66.3\%) showed suicidal ideation.

\section{Logistic regression analysis}

The results of the univariate and adjusted logistic regression analysis of suicidal ideation are also shown in Tables 1 and 2. Of the background characteristics, $\operatorname{sex}(\mathrm{ORu}=$ 2.277; 95\% CI: $1.730-2.997$ ), age ( $\geq 13$ years: $\mathrm{ORu}=0.743$; 95\% CI: 0.560-0.986), and perceived family financial condition (medium: $\mathrm{ORu}=1.774 ;$ 95\% CI: $1.318-2.389$; very poor/poor: $\mathrm{ORu}=5.678$; $95 \% \mathrm{CI}$ : 2.522-12.781) were significantly associated with suicidal ideation (Table 1 ).

The adjusted regression results showed that IGD $(\mathrm{AOR}=$ 3.089; 95\% CI: 2.100-4.544), clinical insomnia (AOR = 5.751; 95\% CI: 3.614-9.152), and presence of depression $(\mathrm{AOR}=14.805 ; 95 \% \mathrm{CI}: 9.685-22.630)$ were all positively associated with suicidal ideation $(p<0.001)$ (Table 2).

\section{Pairwise correlation analysis}

Table 3 shows the results of the pairwise correlation analysis. There were positive and significant correlations among IGD, insomnia, depression, and suicidal ideation $(p<0.001)$.

\section{Path analysis}

The proposed mediation model showed an acceptable goodness of fit $(\mathrm{CFI}=0.974 ; \mathrm{TLI}=0.901 ; \mathrm{RMSEA}=$ 0.076; SRMR $=0.054$ ).
As shown in Table 4 and Fig. 1, there was a significant total effect (standardized estimate [Std. estimate] $=0.341$, $p<0.001$ ) of IGD on suicidal ideation. The direct effect of IGD on suicidal ideation was also significant (Std. estimate $=0.186, p<0.001$ ). In addition, the indirect effects of IGD on suicidal ideation through depression (Std. estimate $=0.083, p<0.001)$ and through insomnia then depression (Std. estimate $=0.053, p<0.001$ ) were significant. However, the indirect effect through insomnia was not significant $(p=0.065)$. Overall, the mediating effect of insomnia and depression were $45.5 \%(0.155 / 0.341$ [Std. estimate of total indirect effect/Std. estimate of total effect]) in the pathway from IGD to suicidal ideation. Moreover, the mediating effect of insomnia accounted for 39.0\% (0.135/0.346 [Std. estimate of indirect effect/Std. estimate of total effect]) of the association between IGD and depression.

\section{Discussion}

This study not only provides regional prevalence data for suicidal ideation, IGD, insomnia, and depression among adolescent Internet gamers in Shanghai, China, but also elucidates the relationships and the underlying mechanisms of these four psychological disorders using a serial multiple mediation model. The prevalence for suicidal ideation in our general study samples and the specific IGD group was 27.2 and $46.9 \%$, respectively. Compared with adolescents with normal Internet use, individuals with IGD were more vulnerable to suicidal ideation. These results accord with those of a previous study of 9758 students from five European countries [43], which found that $45.86 \%$ of students with problematic Internet gaming behaviors showed suicidality. Adolescents with other types of Internet addiction also show a substantial risk of suicidal ideation [18]. Female, 
Table 3 Questionnaire scores and pairwise correlation analysis $(N=1066)$

\begin{tabular}{lllll}
\hline & IGD scale score & ISI scale score & PHQ-9 scale score & Suicidal ideation \\
\hline IGD & 1 & & \\
ISI scale score & $0.240^{* * *}$ & 1 & 1 \\
PHQ-9 scale score & $0.338^{* * *}$ & $0.630^{* * *}$ & $0.509^{* * *}$ & 1 \\
Suicidal ideation & $0.273^{* * *}$ & $0.343^{* * *}$ & \\
\hline
\end{tabular}

IGD Internet gaming disorder, ISI Insomnia Severity Index, PHQ-9 9-item Patient Health Questionnaire $p<0.001$

younger, poorer students in our study showed a greater likelihood of suicidal ideation. These sociodemographic differences echo other findings from different countries $[44,45]$. One study reported that participants with a median age of 13 years (IQR: 8-15 years) [6] showed harmful consequences of suicidal ideation and suicidal attempts that required clinical treatment.

The present findings identified significant relationships among IGD, insomnia, depression, and suicidal ideation, and strongly suggest that more efforts are needed to

Table 4 Results of path analysis $(N=1066)$

\begin{tabular}{|c|c|c|c|c|c|c|c|}
\hline \multirow[t]{2}{*}{ Paths } & \multirow{2}{*}{$\begin{array}{l}\text { Std. } \\
\text { Estimate }\end{array}$} & \multirow[t]{2}{*}{ Estimate } & \multicolumn{2}{|c|}{ Bootstrapping $95 \% \mathrm{Cl}$} & \multirow[t]{2}{*}{ S.E. } & \multirow{2}{*}{$\begin{array}{l}\text { Est./ } \\
\text { S.E. }\end{array}$} & \multirow{2}{*}{$\begin{array}{l}P \\
\text { value }\end{array}$} \\
\hline & & & Lower $2.5 \%$ & Upper $2.5 \%$ & & & \\
\hline \multicolumn{8}{|l|}{ Depression } \\
\hline \multicolumn{8}{|l|}{ Effect } \\
\hline Total effect & 0.346 & 0.850 & 0.688 & 1.018 & 0.085 & 10.012 & $<0.001$ \\
\hline \multicolumn{8}{|l|}{ Direct effect } \\
\hline IGD $\rightarrow$ Depression & 0.211 & 0.518 & 0.382 & 0.658 & 0.071 & 7.268 & $<0.001$ \\
\hline \multicolumn{8}{|l|}{ Indirect effect } \\
\hline IGD $\rightarrow$ Insomnia $\rightarrow$ Depression & 0.135 & 0.332 & 0.249 & 0.423 & 0.044 & 7.476 & $<0.001$ \\
\hline \multicolumn{8}{|l|}{ Suicidal Ideation } \\
\hline \multicolumn{8}{|l|}{ Effect } \\
\hline Total effect & 0.341 & 0.187 & 0.145 & 0.227 & 0.021 & 9.044 & $<0.001$ \\
\hline \multicolumn{8}{|l|}{ Direct effect } \\
\hline IGD $\rightarrow$ Suicidal ideation & 0.186 & 0.102 & 0.062 & 0.141 & 0.020 & 5.061 & $<0.001$ \\
\hline \multicolumn{8}{|l|}{ Indirect effect } \\
\hline Total indirect effect & 0.155 & 0.085 & 0.065 & 0.105 & 0.010 & 8.130 & $<0.001$ \\
\hline \multicolumn{8}{|l|}{ Specific indirect effect } \\
\hline IGD $\rightarrow$ Insomnia $\rightarrow$ Suicidal ideation & 0.018 & 0.010 & 0.000 & 0.021 & 0.005 & 1.849 & 0.065 \\
\hline IGD $\rightarrow$ Depression $\rightarrow$ Suicidal ideation & 0.083 & 0.046 & 0.032 & 0.062 & 0.008 & 5.846 & $<0.001$ \\
\hline IGD $\rightarrow$ Insomnia $\rightarrow$ Depression $\rightarrow$ Suicidal ideation & 0.053 & 0.029 & 0.020 & 0.040 & 0.005 & 5.927 & $<0.001$ \\
\hline \multicolumn{8}{|l|}{ Coefficient } \\
\hline IGD $\rightarrow$ Insomnia & 0.244 & 0.611 & 0.461 & 0.761 & 0.077 & 7.919 & $<0.001$ \\
\hline Insomnia $\rightarrow$ Depression & 0.552 & 0.543 & 0.482 & 0.604 & 0.031 & 17.771 & $<0.001$ \\
\hline Depression $\rightarrow$ Suicidal ideation & 0.396 & 0.088 & 0.071 & 0.104 & 0.009 & 10.148 & $<0.001$ \\
\hline \multicolumn{8}{|l|}{ Insomnia $\rightarrow$ Suicidal ideation } \\
\hline \multicolumn{8}{|l|}{ Effect } \\
\hline Total effect & 0.293 & 0.064 & 0.049 & 0.078 & 0.007 & 8.936 & $<0.001$ \\
\hline Direct effect & 0.074 & 0.016 & 0.000 & 0.033 & 0.008 & 1.919 & 0.055 \\
\hline \multicolumn{8}{|l|}{ Indirect effect } \\
\hline Insomnia $\rightarrow$ Depression $\rightarrow$ Suicidal ideation & 0.219 & 0.048 & 0.038 & 0.058 & 0.005 & 9.045 & $<0.001$ \\
\hline
\end{tabular}

IGD Internet gaming disorder

Std. Estimate (Est.) Standardized estimate, S.E Standard error, $\mathrm{Cl}$ Confidence interval

$p<0.05$ was considered significant 


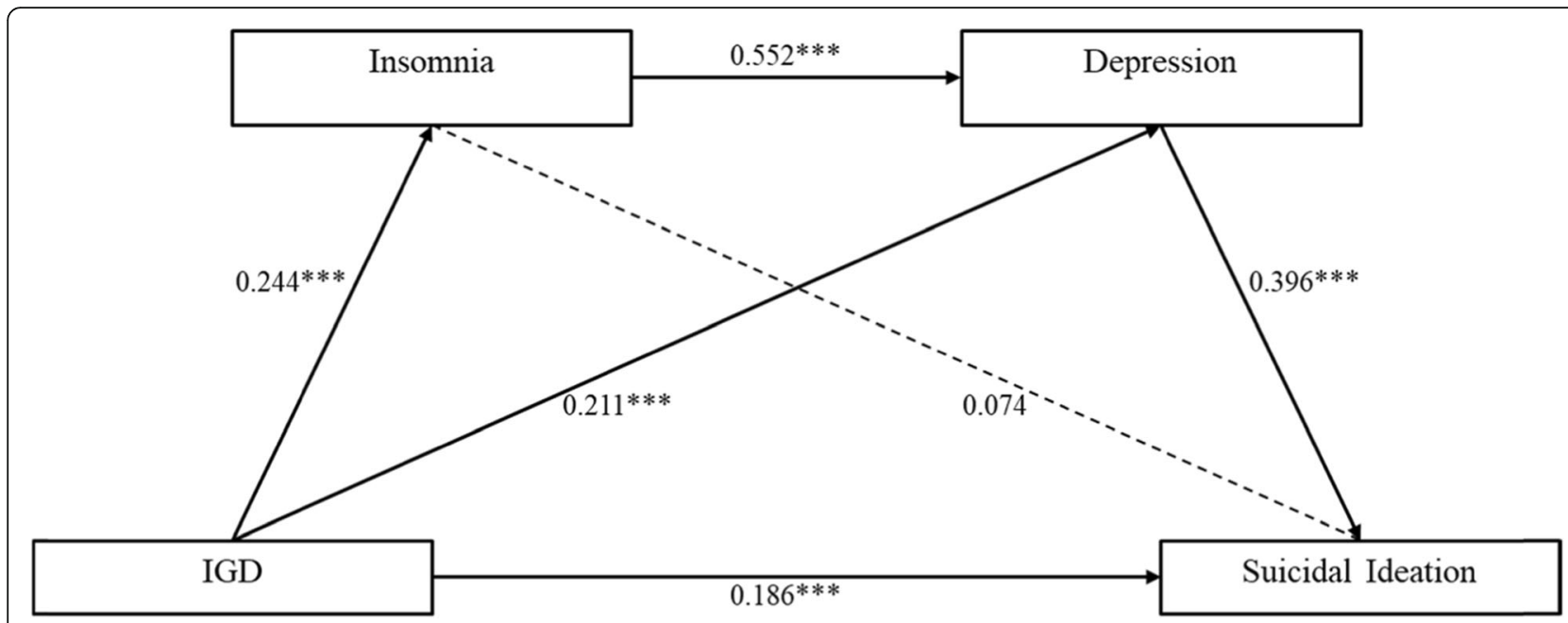

Fig. 1 Path analysis of IGD, insomnia, depression, and suicidal ideation among adolescents ( $N=1066)$ Note: IGD: Internet gaming disorder. Sex, age, and perceived family financial condition were significant in the regression analysis and were adjusted as covariates in the path analysis. Parameters displayed are standardized estimates of the direct effect on each pathway. ${ }^{* *} p<0.001$

monitor Internet use and prevent adolescent insomnia and depression to mitigate the risk of suicidal ideation. Our results show a strong mediating effect $(39.0 \%)$ of insomnia on the association between IGD and depression. This effect is similar to that found in studies conducted with Hong Kong adolescents [46] and adolescents in Nepal [29], yet much lower than the effect (60.6\%) shown for adolescents in Guangzhou, China [27]. This large difference may partly reflect the study population. Although both studies targeted secondary school students, the Guangzhou study included all grades of compulsive education, whereas our study examined only the first grade. The former population may have been exposed to greater sleep deprivation owing to the stress of competing for entrance to senior high schools. Barley et al. [47] emphasized that stress is an important influence on the comorbidity of Internet addiction and insomnia. Thus, the relationships among addiction to types of Internet usage other than IGD, and insomnia and depression need to be further explored while considering the influence of different population background characteristics. Besides, our model tested the bidirectional relationship between IGD and insomnia with a statistically insignificant result, and founded that insomnia was a stronger mediator than IGD in the associations among IGD, insomnia and depression, which was in accordance with previous literature.

This is the first study to examine a serial multiple mediation model of the associations between IGD, insomnia, depression, and suicidal ideation in Chinese adolescents. The mediation model demonstrated that IGD was sequentially correlated with insomnia in the first step, and further positively affected the onset of depression, which was associated with a greater risk of suicidal ideation. Additionally, insomnia alone failed to significantly mediate the pathway to suicidal ideation, which highlights the role of depression as a key mediator in the whole model. This finding is in accordance with a study by Sami et al. [48] on the correlation between sleep disturbance and suicidal ideation. It is possible that depression is the strongest risk factor for suicidal ideation in the presence of IGD, insomnia, or other psychological disorders. It is hard to predict suicidal ideation, but the risk factors identified in this study can be measured. Therefore, better treatment for IGD, insomnia, and depression is central to the prevention of suicidal ideation in the Internet era.

This study had some limitations. First, to reduce the questionnaire length and response time, the suicidal ideation measure was a single question with three response options, which is inadequate for accurate diagnoses. A validated theory-based scale is preferable for identifying suicidal ideation in adolescents. Although many other studies $[49,50]$ have also used a single question (i.e., the ninth item on the PHQ-9 scale, "thoughts that you would be better off dead or of hurting yourself in some way") to identify suicidality, which was similar to the question used here, a more comprehensive inquiry about suicidal ideation would increase the reliability of outcomes. Second, this was a cross-sectional study, and so the reliability of the findings may be low. Longitudinal studies are needed to confirm a temporal effect of these associations. Third, convenience sampling was used to obtain the study population, so the prevalence of suicidal ideation shown here may not apply to adolescents in other regions, as regional differences may affect prevalence. Finally, in addition to the two mediators tested here, other factors may be important in the pathway from IGD to suicidal ideation. For example, impulsivity is a characteristic trait of adolescence and is strongly 
associated with addictive disorders (e.g., IGD) and other risky behaviors (e.g., suicidal ideation) [51]. As adolescence is characterized by internal psychological change and external interpersonal adaption, future studies should examine other psychological factors that may be associated with suicidal ideation.

\section{Conclusions}

This study is the first to explore the relationships among suicidal ideation, IGD, insomnia, and depression in Chinese adolescents. We found a serial multiple mediation effect of insomnia and depression on the pathway from IGD to suicidal ideation. Insomnia first played a partial mediating role in the association between IGD and depression, then depression in turn fully mediated the pathway from insomnia to suicidal ideation. We recommend that interventions for IGD, insomnia, and depression should be strengthened to prevent suicide among adolescents in China.

\section{Abbreviations}

IGD: Internet gaming disorder; ISI: Insomnia Severity Index; PHQ-9: 9-item Patient Health Questionnaire; IQR: Interquartile range; Std. estimate: Standardized estimate; S.E.: Standard error; ORu: Univariate odds ratio; $\mathrm{AOR}$ : Adjusted odds ratio; $\mathrm{Cl}$ : Confidence interval

\section{Acknowledgments}

We would like to thank the participants for their contributions to this study. We thank Diane Williams, PhD, from Liwen Bianji, Edanz Editing China (www. liwenbianji.cn/ac), for editing the English text of a draft of this manuscript.

\section{Authors' contributions}

JTL, YC had the idea for and designed the study and had full access to all data in the study and take responsibility for the integrity of the data and the accuracy of the data analysis. JTL, YC, YY, XY contributed to writing of the manuscript. JTL, YC, YY, XY, SW, HW, RC, LT, SZ, CX, XY contributed to critical revision of the report. $Y C, Y Y$ contributed to the statistical analysis. All authors contributed to data acquisition, data analysis, or data interpretation, and reviewed and approved the final version.

\section{Funding}

This work was supported by the National Natural Science Foundation of China [grant numbers 71673187, 81373021]; the National Key Research and Development Project [grant numbers 2018YFC1705100, 2018YFC1705103]; Shanghai Three-year Action Plan for Public Health [grant numbers GWV-10.1XK15, GW-10.1-XK18, GW-10.2-XD13]. The funding body played no role in the design of the study and collection, analysis, and interpretation of data and in writing the manuscript.

\section{Availability of data and materials}

Data and materials used in this study are available from the corresponding authors and will be made available on reasonable request.

\section{Ethics approval and consent to participate}

The whole research process strictly complied with American Psychological Association ethical standards and with the Code of Ethics of the World Medical Association (Declaration of Helsinki). Verbal informed consent was obtained from all participants and their legal guardians before data collection, considering that the investigation was conducted during school time thus the school principals were responsible for them. The ethics committee for our study approved this method of obtaining consent. The study protocol and procedure were approved by the Survey and Behavioral Research Ethics Committee of the Chinese University of Hong Kong.
Consent for publication

Not applicable.

\section{Competing interests}

The authors declare that they have no competing interests.

Received: 5 June 2020 Accepted: 15 September 2020

Published online: 23 September 2020

\section{References}

1. Organization WH. Disease and injury country mortality estimates, 2000-2015 [Data files]. Retrieved September 23. 2017.

2. Hawton K, Bergen H, Kapur N, Cooper J, Steeg S, Ness J, et al. Repetition of self-harm and suicide following self-harm in children and adolescents: findings from the Multicentre Study of Self-harm in England. J Child Psychol Psychiatry. 2012;53(12):1212-9.

3. Cluver L, Orkin M, Boyes ME, Sherr L. Child and adolescent suicide attempts, suicidal behavior, and adverse childhood experiences in South Africa: a prospective study. J Adolesc Health. 2015;57(1):52-9.

4. Lim KS, Wong CH, Mclntyre RS, Wang J, Zhang Z, Tran BX, et al. Global Lifetime and 12-Month Prevalence of Suicidal Behavior, Deliberate Self-Harm and Non-Suicidal Self-Injury in Children and Adolescents between 1989 and 2018: A Meta-Analysis. Int J Environ Res Public Health. 2019;16(22):4581.

5. Kwok S, Gu M, Cheung A. A longitudinal study on the relationship among childhood emotional abuse, gratitude, and suicidal ideation of Chinese adolescents. Child Abuse Negl. 2019;94:104031.

6. Burstein B, Agostino H, Greenfield B. Suicidal attempts and ideation among children and adolescents in US emergency departments, 2007-2015. JAMA Pediatr. 2019;173(6):598-600.

7. Organization WH. The ICD-11 classification of mental and behavioral disorders: diagnostic criteria for research. Geneva: World Health Organization; 2018.

8. Petry NM, Rehbein F, Gentile DA, Lemmens JS, Rumpf H-J, Mößle T, et al. An international consensus for assessing internet gaming disorder using the new DSM-5 approach. Addiction. 2014;109(9):1399-406.

9. Fam JY. Prevalence of internet gaming disorder in adolescents: a metaanalysis across three decades. Scand J Psychol. 2018;59(5):524-31..

10. Chen YL, Gau SS. Sleep problems and internet addiction among children and adolescents: a longitudinal study. J Sleep Res. 2016;25(4):458-65.

11. Alimoradi Z, Lin CY, Broström A, Bülow PH, Bajalan Z, Griffiths MD, et al. Internet addiction and sleep problems: A systematic review and metaanalysis. Sleep Med Rev. 2019;47:51-61.

12. Liu M, Wu L, Yao S. Dose-response association of screen time-based sedentary behaviour in children and adolescents and depression: a metaanalysis of observational studies. Br J Sports Med. 2016;50(20):1252-8.

13. Strong C, Lee CT, Chao LH, Lin CY, Tsai MC. Adolescent internet use, social integration, and depressive symptoms: analysis from a longitudinal cohort survey. J Dev Behav Pediatr. 2018;39(4):318-24.

14. VANR AJ, Kuss DJ, Griffiths MD, Shorter GW, Schoenmakers MT, VDM D. The (co-)occurrence of problematic video gaming, substance use, and psychosocial problems in adolescents. J Behav Addict. 2014;3(3):157-65.

15. Pan PY, Yeh CB. Internet addiction among adolescents may predict selfharm/suicidal behavior: a prospective study. J Pediatr. 2018;197:262-7.

16. Lee SY, Park EC, Han KT, Kim SJ, Chun SY, Park S. The Association of Level of internet use with suicidal ideation and suicide attempts in south Korean adolescents: a focus on family structure and household economic status. Can J Psychiatr. 2016;61(4):243-51.

17. Lin $\mathrm{H}$, Ko C-H, Chang Y-P, Liu T-L, Wang P-W, Lin H-C, et al. The association between suicidality and internet addiction and activities in Taiwanese adolescents. Compr Psychiatry. 2014;55(3):504-10.

18. Cheng YS, Tseng PT, Lin PY, Chen TY, Stubbs B, Carvalho AF, et al. Internet Addiction and Its Relationship With Suicidal Behaviors: A Meta-Analysis of Multinational Observational Studies. J Clin Psychiatry. 2018;79(4):17r11761.

19. Messias E, Castro J, Saini A, Usman M, Peeples D. Sadness, suicide, and their association with video game and internet overuse among teens: results from the youth risk behavior survey 2007 and 2009. Suicide Life Threat Behav. 2011:41(3):307-15.

20. Kang N, You J, Huang J, Ren Y, Lin MP, Xu S. Understanding the pathways from depression to suicidal risk from the perspective of the interpersonalpsychological theory of suicide. Suicide Life Threat Behav. 2019;49(3):684-94. 
21. Cavanagh JT, Carson AJ, Sharpe M, Lawrie SM. Psychological autopsy studies of suicide: a systematic review. Psychol Med. 2003;33(3):395-405

22. Jen-Wei L, Yu-Kang T, Ying-Fan L, Hsin-Chien L, Pei-Shan T, Ting-Jhen C, et al. Associations between sleep disturbances and suicidal ideation, plans, and attempts in adolescents: a systematic review and meta-analysis. Sleep. 2019;6:6.

23. Lam LT. Internet gaming addiction, problematic use of the internet, and sleep problems: a systematic review. Curr Psychiatry Rep. 2014;16(4):444.

24. Long Z, Yi Z, Dongfeng Z. Sedentary behaviour and the risk of depression: a meta-analysis. Bri J Sports Med. 2015;49(11):705-9.

25. Chen R, Liu J, Cao X, Duan S, Wen S, Zhang S, et al. The relationship between mobile phone use and suicide-related behaviors among adolescents: the mediating role of depression and interpersonal problems. Affect Disord. 2020;269:101-7.

26. Li L, Wu C, Gan Y, Qu X, Lu Z. Insomnia and the risk of depression: a metaanalysis of prospective cohort studies. BMC Psychiatry. 2016;16(1):375.

27. Li JB, Lau JTF, Mo PKH, Su XF, Tang J, Qin ZG, et al. Insomnia partially mediated the association between problematic internet use and depression among secondary school students in China. J Behav Addict. 2017;6(4):554-63.

28. Vernon L, Modecki KL, Barber BL. Tracking Effects of Problematic Social Networking on Adolescent Psychopathology: The Mediating Role of Sleep Disruptions. J Clin Child Adolesc Psychol. 2017;46(2):269-83.

29. Bhandari PM, Neupane D, Rijal S, Thapa K, Mishra SR, Poudyal AK. Sleep quality, internet addiction and depressive symptoms among undergraduate students in Nepal. BMC Psychiatry. 2017;17(1):106.

30. Association APA. Diagnostic and Statistical Manual of Mental Disorders: DSM-V1994.

31. Morin CM, Press TG. Insomnia: Psychological Assessment and Management 1993(6).

32. Bastien $\mathrm{CH}$, Vallières $\mathrm{A}$, Morin $\mathrm{CM}$. Validation of the insomnia severity index as an outcome measure for insomnia research. Sleep Med. 2001;2(4):297-307.

33. Chahoud M, Chahine R, Salameh P, Sauleau E. A. Reliability, factor analysis and internal consistency calculation of the insomnia severity index (ISI) in French and in English among Lebanese adolescents. eNeurologicalSci. 2017; 7:9-14.

34. Badiee ABS, Torabi S, Gao Y, Cao KG, Badiee ABHR. P03-386 - Relaibility and validity of the Chinese translation of insomnia severity index (C-ISI) in Chinese patients with insomnia. Eur Psychiatry. 2011;26(Suppl 1):1556.

35. Gagnon $C$, Bélanger $L$, Ivers $H$, Morin CM. Validation of the insomnia severity index in primary care. J Am Board Fam Med. 2013;26(6):701-10.

36. Kroenke K, Spitzer RL, JB W. The PHQ-9 : Validity of a Brief Depression Severity Measure. J Gen Intern Med. 2001;16(9):606-13.

37. Wang W, Bian Q, Zhao Y, Li X, Wang W, Du J, et al. Reliability and validity of the Chinese version of the Patient Health Questionnaire (PHQ-9) in the general population. Gen Hosp Psychiatry. 2014;36(5):539-44

38. Manea L, Gilbody S, Mcmillan D. A diagnostic meta-analysis of the patient health questionnaire-9 (phq-9) algorithm scoring method as a screen for depression. Gen Hosp Psychiatry. 2015;37(1):67-75.

39. Muñoz-Navarro R, Cano-Vindel A, Medrano LA, Schmitz F, Ruiz-Rodríguez P, Abellán-Maeso C, et al. Utility of the PHQ-9 to identify major depressive disorder in adult patients in Spanish primary care centres. BMC Psychiatry. 2017;17(1):291.

40. Kristopher J, Preacher AFH. Asymptotic and resampling strategies for assessing and comparing indirect effects in multiple mediator models. Behav Res Methods. 2008;40(3):879-91.

41. Wen Z, Ye B. Analyses of mediating effects: the development of methods and models (in Chinese with an English abstract). Adv Psychol Sci. 2014; 22(5):731-45.

42. Mcdonald RP, Moon-Ho RH. Principles and practice in reporting structural equation analyses. Psychol Methods. 2002;7(1):64.

43. Strittmatter E, Kaess M, Parzer P, Fischer G, Carli V, Hoven CW, et al. Pathological internet use among adolescents: comparing gamers and nongamers. Psychiatry Res. 2015;228(1):128-35.

44. Kann L, McManus T, Harris WA, Shanklin SL, Flint KH, Queen B, et al. Youth Risk Behavior Surveillance - United States, 2017. Morbidity and mortality weekly report Surveillance summaries (Washington, DC). 2018;67(8):1-114.

45. Zhang YY, Lei YT, Song Y, Lu RR, Duan JL, Prochaska JJ. Gender differences in suicidal ideation and health-risk behaviors among high school students in Beijing, China. J Glob Health. 2019;9(1):010604.

46. Cheung LM, Wong WS. The effects of insomnia and internet addiction on depression in Hong Kong Chinese adolescents: an exploratory crosssectional analysis. J Sleep Res. 2011;20(2):311-7.
47. Barley SR, Meyerson DE, Grodal S. E-mail as a Source and Symbol of Stress 2011;22(4):887-906.

48. Sami H, Danielle L, Lihi D, Elena S. The effect of sleep disturbances and internet addiction on suicidal ideation among adolescents in the presence of depressive symptoms. Psychiatry Res. 2018;267:327-32.

49. Uebelacker LA, German NM, Gaudiano BA, Miller IW. Patient health questionnaire depression scale as a suicide screening instrument in depressed primary care patients: a cross-sectional study. Prim Care Companion CNS Disord. 2011:13(1):PCC.10m01027.

50. Bauer AM, Chan Y-F, Huang H, Vannoy S, Unützer J. Characteristics, management, and depression outcomes of primary care patients who endorse thoughts of death or suicide on the PHQ-9. J Gen Intern Med. 2013;28(3):363-9.

51. Auerbach RP, Stewart JG, Johnson SL. Impulsivity and Suicidality in adolescent inpatients. J Abnorm Child Psychol. 2017:45(1):91-103.

\section{Publisher's Note}

Springer Nature remains neutral with regard to jurisdictional claims in published maps and institutional affiliations.
Ready to submit your research? Choose BMC and benefit from:

- fast, convenient online submission

- thorough peer review by experienced researchers in your field

- rapid publication on acceptance

- support for research data, including large and complex data types

- gold Open Access which fosters wider collaboration and increased citations

- maximum visibility for your research: over $100 \mathrm{M}$ website views per year

At BMC, research is always in progress.

Learn more biomedcentral.com/submissions 